\title{
Prospective benefits and criticalities of applying Semantic Web techniques in Software Engineering
}

\author{
Harvinder \\ Asst. Professor, Galgotias College of Engineering and Technology \\ Email: me.harvinder@gmail.com
}

\begin{abstract}
:
The technology is getting advanced day by day and so do the softwares. To manage softwares effectively one needs to use the concepts of software engineering efficiently and the concept of Semantic web can be used to reuse the existing softwares. Domain of software engineering is very large and there are numerous engineers working on ample amount of software products. The products are different in their terminology, interface, and look \& feel. One thing that is common is that similar logics are being applied to various products. Using Semantic web, the product having similar logics can be translated into Ontologies. These Ontologies can be further used to make efficient reuse of Softwares. This paper proposes both the advantages as well as criticalities involved while using Semantic Web for Softwares. On one hand it can be observed that using semantics can save a lot of time and effort and on other technically various hurdles can come on the way.
\end{abstract}

Keywords: Semantic web, Software Engineering, Ontologies

\section{Introduction}

Globalization has hugely increased from last few years. Earlier people lived at their own country, ate their own staple food and spoke their own language. Similar is the case of computers, earlier machines used to be environment specific and similar kind of work was done on them, But with globalization both scenarios have changed hugely. Computers have drastically improved the way information used to be before some years. It hardly takes fractions of seconds to make an information reach in any part of the world. The fact now is you can get whatever information you want in a very little time anywhere in the world, but till now the search scenario is not capable to respond each and every request efficiently. The internet boom has electrified the world and changed the way world looked .

Various advancements have been done, but till now a user can only search for some data and has to derive information on his/ her own. Technically speaking, "A computer has a lot of memory but no brain". Basic idea of Semantic web that machines must be capable of retrieving the right 
kind of information from the given data and respond with the correct result and all that without human intervention.
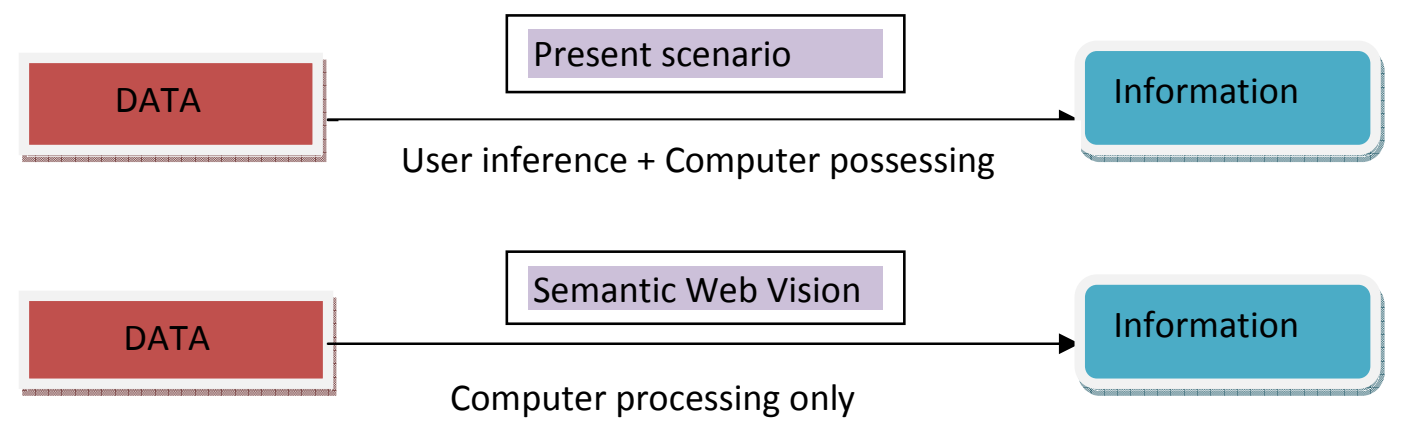

Figure1. Scenario before and after full implementation of Semantics in web

Regarding the searching scenario, we began with the keyword search, and then rank based search happened. If we observe closely both kinds of searches are not appropriate. For example, if a person search "lowest airfare airline from Delhi to Mumbai", the response you'll get is various websites claiming to be the lowest. But the ultimate conclusion or lowest airfare can be deciphered by you only after watching all those sites(supposed to be lowest airfare offering sites).Hence, the technologies like crawlers ,spiders, screen scrappers etc can definitely make the task easy but ultimately they do not understand "what do you mean?'This is the problem for which continuously technologies of Semantic web are being proposed.

The fragility of software lies in the fact that it cannot understand the content and can't combine knowledge \& experience to give useful result.

In short it can be said that, Today's web limitations are:

i) We search for hours and we might not find anything relevant.

ii) Help is limited only to display of a page.

To overcome these, the idea of semantic web is to make software understandable content, which can be used by machines for processing. The idea of software is to tell meaning and relations of each and every term. Slowly, billions of convergence and assertions will lead to conclusion that is same as real world.

\section{Semantic Web}

The Semantic Web is a mesh of information linked up in such a way as to be easily processable by machines, on a global scale. It can be thought as being an efficient way of representing data on the internet or simply termed as worldwide linked database. [1]

Abundant information is available now a day's via the internet leading to efforts to enhance the usefulness, efficiency and selectivity of a variety of search engines. The World Wide Web is possible only because of a set of broadly established standards that guarantee interoperability at various levels. Till now, the Web has been intended for direct human processing, but the nextgeneration Web called the "Semantic Web", aims at machine- processable information. The word "Semantic" refers to "meaning" - a semantic web is actually a web of meaning .It will be referred as a web that identifies what the entities on the web, what do those mean and make use of that information proficiently. [2] The Semantic Web will enable intelligent services, which 
offer greater functionality and interoperability than current stand-alone services. It can only become possible if once further levels of interoperability have been established. Various standards will have to be defined for the exact description of semantic content of the data. For the semantic web to function properly and efficiently, computers must have an access to structured collections of information and sets of information and sets of inference rules that they can use to conduct automated reasoning Reference rules that they can use to carry out automated reasoning.

The aim of the semantic web is to make the WWW agents friendlier than their present scenario. By adding semantic information to Web, we can augment the effectiveness and selectivity of search engines and other automated document processing tools. The Semantic Web facilitates computers to seek information that is distributed throughout the Web, mesh it, and then take act according to the information received. Resource Description Framework (RDF) is the world wide web consortium's standard for encoding knowledge which is distributed throughout. [3] The knowledge available on the current web is very limited in scope and usage. For example a Web page, which may communicate a lot of information to the human reader, but to the machine which is displaying the page all it sees, is presentation markup. To computers these are just pages of HTML, images, Flash, etc. The knowledge the files are conveying to the human is absolutely opaque to the computers, which are displaying that information. The "semantic" in Semantic Web is not that computers are going to understand the meaning of anything written in various documents, but it simply mean that the logical pieces of meaning can be manipulated by a machine to useful human ends. So, imagine a new web where the actual content of internet can be manipulated and modified by computers which were till now only displaying the content. It can also be seen as web of databases where the data is stored throughout the world.

As an example, suppose one semantic website distributes a database about a product series, with its products and descriptions, while a second publishes a database of product reviews. A third site for a retailer publishes a database of products in stock. Some standards can make it easier to write an application to mesh dispersed databases together, so that a computer could use the three data sources together to help an end user make improved purchasing decisions.

\section{Ontologies}

The history of artificial intelligence shows that knowledge is critical for intelligent systems. In many cases, better knowledge can be more important for solving a task than better algorithms. To have truly intelligent systems, knowledge needs to be captured, processed, reused, and communicated. Ontologies support all these tasks.

The term "ontology" can be defined as an explicit specification of conceptualization. Ontologies confine the structure of any given domain, i.e. conceptualization. This will comprise of the model of the domain having many possible limitations. This conceptualization depicts facts about whole of the domain and not about a particular state of affair in a given domain. Also we can say that, the conceptualization will not change, some concepts might change but not whole of the basic form. Ontology is that specification of conceptualization - the conceptualization which is specified by using a particular modeling language, particular model or some particular terms related o one concept only. Formal specification is necessary so that processing of ontologies can be done easily and further they can work independently and automatically. 
Ontology is used to describe a domain, while a knowledge base (based on ontology) is used to describe a particular status of affairs. Both of them together will be helpful to create a large database which can be used to access the processes throughout the world. [4]

\section{Semantic Web and Software Engineering}

Software engineering is the "application of a systematic, disciplined, quantifiable approach to the development, operation, and maintenance of software" [5] and Semantic Web is a "manmade woven web of data" that facilitates machines to understand the semantics, or meaning, of information on the World Wide Web.[6][7]

Software development in itself is a huge process and include ample amount of time and effort. If used and operated wisely and efficiently, lots of efforts can be saved. The fact that various processes, designs and codes etc can be reused and globalized easily once ontologies are designed, cannot be ignored.

Using semantic web with software engineering techniques majorly look at the problems in single domain. There are lots of proposals to include Ontologies in various stages of Software Development Life Cycle (SDLC). The main purpose of the Ontologies and Semantic web is to put together heterogeneous data and thus enable the interoperability among various disparate systems present on internet. The term Ontology can be used to model software engineering knowledge by representing the artifacts that are premeditated or formed during whole of the SDLC (Software Development Lifecycle) process. The Semantic Web allows publishing of reusable software engineering resources of knowledge and providing numerous services for querying and further searching. It is a well known fact that Software development is a complex process and it generate a very large quantity of information. Thus, a well, disciplined, systematic and quantifiable approaches are required for the operation, development and maintenance of software systems [8].

Software Engineering discipline is one of the vastest in the field of software from the time it exists. This community keeps on developing various tools for development, maintenance and updating of Software Engineering tools. Majorly All the tools use the predefined prototypes in order to develop new applications. This pre definition of formats sometimes causes the problem of interoperability. Numerous efforts have been made to improve the software processes. For example, various modeling languages have been proposed to represent system designs in a better way, the Integrated Development Environments (IDEs) have been formed to facilitate implementation in an efficient manner. Also, various types of Computer-Aided Software Engineering (CASE) tools have been developed to manage different types of software artifacts. Despite all these, software development still remains tricky in numerous ways. Any software process involves a lot of efforts in gaining and producing given set of information. Undoubtedly, reusing existing information saves lot of time and efforts. The reuse scope has been expanded now from reusing code pieces to reusing all other kinds of information, s.a requirements, and designs, various project processes, and software implementations. The representation of all these artifacts in the current scenario makes it very hard to manage the information followed by its retrieval and reuse. Therefore, a suitable method which can facilitates information retrieval and can promote the reuse is highly demanded. Also due to globalization, software systems are usually developed among geographically dispersed teams.Therefore, diversity definitely exists among the processes used by different teams. Therefore to increase the quality of product, some method is needed to reuse the code and process data efficiently.

The Semantic Web provides a way to improve information is exchanged and interoperability is required. In this paper, only the field of Software Engineering has been covered but the scope of 
Semantic Web are not limited to Software Engineering. Semantic Web can be used to reduce barriers of predefined formats in any domain where the same is required. [9]

\section{Benefits and criticalities of applying Semantic Web techniques in Software Engineering}

\subsection{Advantages:}

\section{a) Globalization of Software products made easier}

There are numerous softwares being programmed all over the world. The idea to introduce common ontologies for all the softwares will make each software understand the terminology of other. Thus it will be easy to use the software globally anywhere and at any platform. Software ontology design is a tedious task but once it is done, user intervention for communication between two softwares will not be required. Thus the idea of machine understandability can easily be united with globalization.

\section{b) Reuse of Software codes}

Software engineering involves various steps to make software. Whole life cycle of the product involves lot of input before getting a fruitful output. The task of designing and implementing software is an effortful task. So, in this scenario it is very useful to reuse the existing set of programs rather to make new ones. Inheritance and reuse are used presently also but the reuse capability is limited to similar kinds of projects only. To efficiently reuse the softwares, the terminology used must be same. This can be done by making RDF triplets \& ontologies. Once it is done appropriately, the extent of reusability will reach a new high.

c) Fusion of softwares may be possible:

Different softwares have different prototypes, different designs and different target customers. It may be possible to fuse two or more softwares based on their ontologies to make new software. Functionalities of two or more softwares can be targeted into one project in order to make hybrid software. This can reduce the effort required to make new software.

\section{d) Cross Domain communication:}

Domain Engineers can make different ontologies for different domains. Some Ontologies like Application domain ontology [10] [1] [4] [8] have earlier been proposed to reuse the softwares that are either of same domain or of different domains.

\section{e) Standard Specification of formal requirements:}

Specification of requirements (during requirement phase) needs gathering the information from the customer. Then these requirements are jotted down formally to make both the parties agree upon. Ontologies may be used to make a semi formal structure which is understood by both the parties (client and designer).So in contrast to traditional way 
formalization of Ontologies may offer a mid-way solution. However, the solution we are referring here can be used only with functional requirements and not with non functional requirements.

\subsection{Criticalities:}

a) Globalization can be boon or ban: It can be either boon or ban depending on the tasks that a person is doing. Writing on paper about global scenario is very easy but to implement it practically is very difficult. In every phase of SDLC desired semantics are a great challenge to achieve. Also existing software models ought to be changed in order to get them abreast with semantics. There can be various hurdles on the way of actual implementation of truly global ontologies.

b) Vocabularies of two Ontologies may vary: Complete process model of any software can only be established after creating mapping between all the terms that share same meaning. In that case, overlapping has to be identified and removed before actually making it a Formal ontology. This rectification of overlapping is a long, time consuming and conscious effort so as to avoid any ambiguity in future. A term used by two different clients may have different meaning or may be two different words used by two parties may have the same meaning. Mapping such terms is another big milestone to be achieved.

c) Functional requirements can be stated while non functional cannot be stated: Functional requirement can be documented according to the user's requirements .Guidelines can be easily framed as per the client's requirements in Software Requirement Specification. Ontology designing is also easy when the requirements are well stated. The problem here rises when the requirements are non functional. Non functional Requirements are majorly related to Quality. The level of Quality depends on a particular client and it cannot be stated in words always. Therefore, making Ontologies of requirements and then to make it same globally is a lengthy and tiresome task.

d) Difficulty in designing Web-Services: Web services are majorly used to retrieve services from Ontologies. Semantic web can only be helpful when the mapping of Ontologies is done properly. Also, change of one service protocol/code may lead to change in whole ontology. To accomplish all the changes in web services may become a cumbersome task

\section{Conclusion}

Fully implemented Semantic web is till now a vision and to fulfill the same various domains have to merge together to successfully giving the answers to the queries generated by various systems. In this paper only the field of software engineering is covered elaborating how semantic web can be beneficial for whole internet searching and surfing.

Semantic web will change the way we work, we see the things and we do things on internet. Other benefits like portability, globalization etc are added advantages. 
Looking at the other side of coin, challenges in implementing ontologies are huge and ought to be handled with great caution. One technically wrong concept can disturb the whole SDLC cycle. Combining the various artifacts can lead to redundancy and ambiguity which must be solved beforehand while designing the ontologies.

Worldwide there are numerous programmers and everyone has his/her own style and norms of programming. To cater for the needs of all will take lot of time and approvals from the higher levels. No matter what and how we implement, the main concern that has to be taken care of is the successful querying of data in machines. Only correct results will solve the real purpose of Semantic Web.

\section{Related work}

In future, various ontologies related to software engineering field can be designed which can be used to club various softwares. Using same ontologies will give a common platform to all softwares.

Onlotlogy designing tools for various phases of software engineering is a major task that has to be done. For example, in requirement phase the ontology can be designed s.t every programmer input the requirement in the same manner. This will help to maintain a common platform amongst all the requirements takes in various softwares. Then in design phase, common tools and methods can be used to design any software worldwide. When any changes need to be done, they can be done by inculcating modifications in ontologies. Next, during implementation phase ontologies might not play major role, as different players do the task differently.

Ontology Designing tools for various phases of SDLC and then making a query language that can cater the needs of various machines is the task that can be done in future.

\section{References:}

[1] Semantic Web introduction (http://infomesh.net/2001/swintro/)

[2] Berners-Lee, T., Hendler J. and Lassila,O: The Semantic Web. Scientific American 284(5)

[3] Introduction to RDF and RDFS ( http://www.xml.com/pub/a/2001/01/24/rdf.html)

[4] Ontologies and Semantic Web (http://www.obitko.com/tutorials/ontologies-semanticweb/ontologies.html)

[5] Abran, A., and Moore, J.W. (Exec. Eds.), Bourque, P. and Dupuis, R. (Eds.) Guide to the Software Engineering Body of Knowledge (2004) 
Advanced Computing: An International Journal ( ACIJ ), Vol.2, No.6, November 2011

[6] World Wide Web Consortium (W3C) Semantic Web activity's homepage. (http://w3c.org/sw.)

[7] The Semantic Web by Eric Miller (http://www.w3c.org)

[8] IEEE Std 610.12-1990, IEEE Standard Glossary of Software Engineering Terminology, IEEE, 1990.

[9] Yajing Zhao, Jing Dong, Senior Member, IEEE, and Tu Peng Ontology Classification for Semantic-Web-Based Software Engineering, IEEE TRANSACTIONS ON SERVICE COMPUTING, VOL. 2, NO. 4, OCTOBER-DECEMBER 2009

[10] H.-J. Happel and S. Seedorf. Applications of ontologies in software engineering.(2nd Workshop on Semantic Web Enabled Software Engineering (SWESE 2006) at ISWC 2006, Galway, Ireland, November 11-15, 2006) 